\title{
IC 361, a distant intermediate-age cluster in Camelopardalis $\dagger$
}

\author{
Justas Zdanavičius, ${ }^{1}$ Richard P. Boyle, ${ }^{2}$ Frederick J. Vrba,${ }^{3}$ \\ Kazimieras Zdanavičius ${ }^{4}$ and Stanislava Bartašiūtè ${ }^{1}$ \\ ${ }^{1}$ Astronomical Observatory of Vilnius University, Čiurlionio 29, Vilnius, LT-03100, Lithuania \\ email: [justas, kz]@itpa.lt \\ ${ }^{2}$ Vatican Observatory Research Group, Steward Observatory, Tucson, AZ 85721, USA \\ email: boyle@ricci.as.arizona.edu \\ ${ }^{3}$ US Naval Observatory Flagstaff Station, P.O. Box 1149, Flagstaff, AZ 86002, USA \\ email: fjv@nofs.navy.mil \\ ${ }^{4}$ Institute of Theoretical Physics and Astronomy, Vilnius University, Goštauto 12, Vilnius, \\ LT-01108, Lithuania
}

\begin{abstract}
We have undertaken CCD photometry in the eight-color Vilnius $+I$ system for the open cluster IC $361\left(l=147.5^{\circ}, b=5.7^{\circ}\right)$ located in the constellation of Camelopardalis. Based on multicolor data, estimates of distance moduli, foreground reddening and metallicity have been obtained for individual stars measured in the field of the cluster. This allowed us to eliminate most of the field stars and to identify the probable cluster members, from which we derived the true distance modulus $(m-M)_{0}=12.7$ mag and metallicity $[\mathrm{Fe} / \mathrm{H}]=-0.3$ dex. The interstellar extinction is found to be nonuniform across the field, with values of $A_{V}$ ranging from 1.9 to 2.6 mag. A comparison of the color-magnitude diagrams with Padova isochrones yields $(m-M)_{0}=12.6 \mathrm{mag}$, or a cluster distance of $3.3 \mathrm{kpc}$, and an age of 1.0 Gyr. Therefore, IC 361 appears to be a mildly metal-deficient cluster of intermediate age, located as far as, or just beyond, the Perseus spiral arm.
\end{abstract}

Keywords. techniques: photometric, open clusters and associations: individual (IC 361)

\section{Introduction}

The open cluster IC 361 is well detached from the field, but because of faintness it has been very poorly studied, if not completely forgotten. The only photometric information published to date is that by Piccirillo \& Stein (1978), who obtained photoelectric $U B V$ and photographic $B V$ data for 19 and 32 stars in the field of the cluster, respectively, and identified the contours of the main sequence and a few red-giant stars. Based on these preliminary data they adopted a distance of $2.5 \mathrm{kpc}$, a high reddening value, $E(B-V)=0.55 \mathrm{mag}$, and suggested the age in the range from 0.5 to 1 Gyr. IC 361 lies in the second Galactic quadrant $\left(\ell=147.5^{\circ}, b=5.7^{\circ}\right)$, in the immediate vicinity of the Camelopardalis dark clouds. Therefore, it is not surprising that the cluster could be subject to considerable interstellar reddening. If so, multicolor intermediate-band photometry can provide improved determinations of the fundamental cluster parameters.

$\dagger$ The full poster (in pdf format) is available at http://www. astro.iag.usp.br/ iaus266/Posters/pZdanavicius.pdf. 

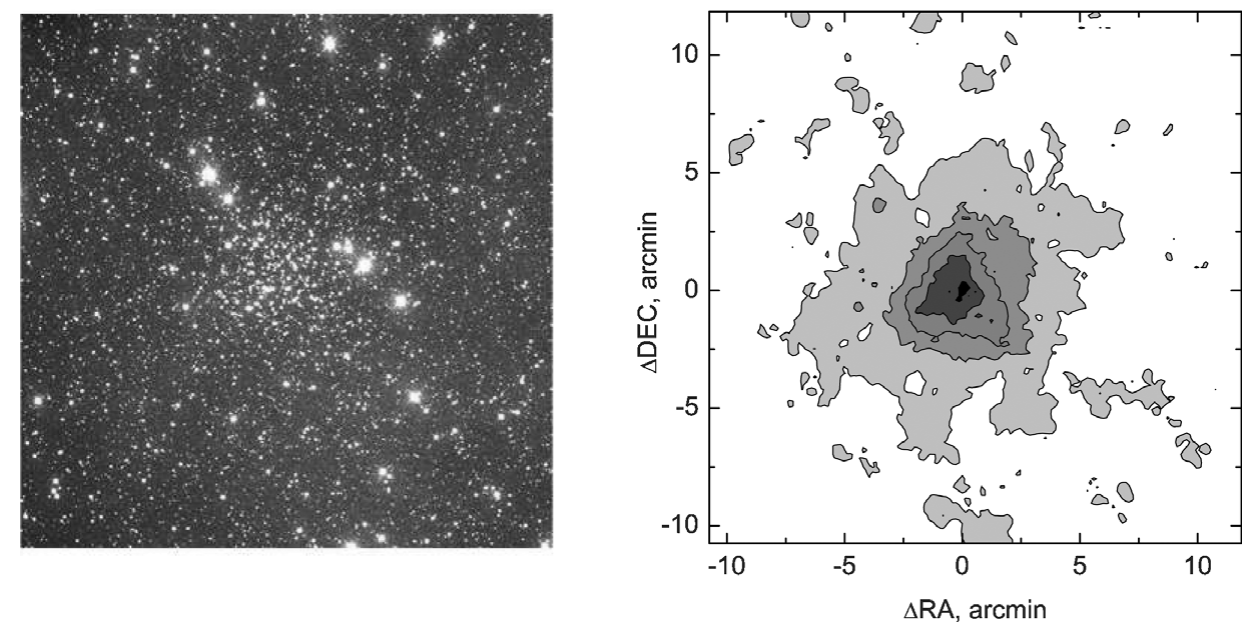

Figure 1. (left) Image of the field of IC $361\left(20^{\prime} \times 20^{\prime}\right)$ in the $I$ filter (Flagstaff $\left.2 \mathrm{~K} \mathrm{CCD}\right)$. (right) Isodensity contours of stars measured in the $I$ filter.

\section{Observations and data reductions}

CCD observations in seven filters (UPXYZVS) of the intermediate-band Vilnius system and in the $I$ band were carried out in 1995 with a $2 \mathrm{~K} \times 2 \mathrm{~K}$ CCD camera on the $1 \mathrm{~m}$ telescope of the USNO Flagstaff Station, giving an unvignetted field of $20^{\prime} \times 20^{\prime}$ (Figure 1, left panel). Additional observations of the central part of the field $\left(12^{\prime} \times 12^{\prime}\right)$ in the Vilnius filters $U Y V$ were obtained in 2008 with a $4 \mathrm{~K}$ CCD camera at the $1.8 \mathrm{~m}$ Vatican Advanced Technology Telescope (VATT) on Mt. Graham (Arizona). Repeated exposures in the UPXYZVS filters were also taken in March 2009 using the same $1 \mathrm{~m}$ telescope of the USNO Flagstaff Station, but these new observations have not yet been included in the present analysis.

The CCD data frames were reduced using the IRAF packages, by combining both the aperture and point-spread-function method. The calibration equations were obtained by observing the standard field of M67 (Laugalys et al. 2004). In the Flagstaff field, a total of 1450 stars brighter than $V=18.5$ mag were measured in seven Vilnius filters, while in the $I$ filter the number of stars measured reaches 7250. With the VATT telescope, a limiting magnitude of $V=19.5 \mathrm{mag}$ was obtained with a good signal-to-noise ratio. The color-magnitude diagrams (CMDs), $V, V-Y$ and $V, V-I$, for stars common to the VATT and Flagstaff data sets are shown in Figure 2.

\section{Results}

The coordinates of the cluster center, corresponding to the density peaks in our photometry, are

$$
\alpha=4^{\mathrm{h}} 18^{\mathrm{m}} 55.0^{\mathrm{s}}, \delta=+58^{\circ} 14^{\prime} 53^{\prime \prime}(2000) .
$$

According to the surface-density distribution of stars measured in the $I$ filter, the cluster has a radius of $7^{\prime}$ and a fairly strong central concentration (Figure 1, right panel).

The classification of stars in terms of spectral class, absolute magnitude and interstellar reddening, $E(Y-V)$, was performed using the reddening-free $Q$ parameters. In calculating the $Q \mathrm{~s}$, we used the color-excess ratios corresponding to the normal interstellar extinction law. Since we applied several classification methods, such as SIGmaQ, COMPAR, XQKLAS, and XDX, the values derived from an average of these classifications 

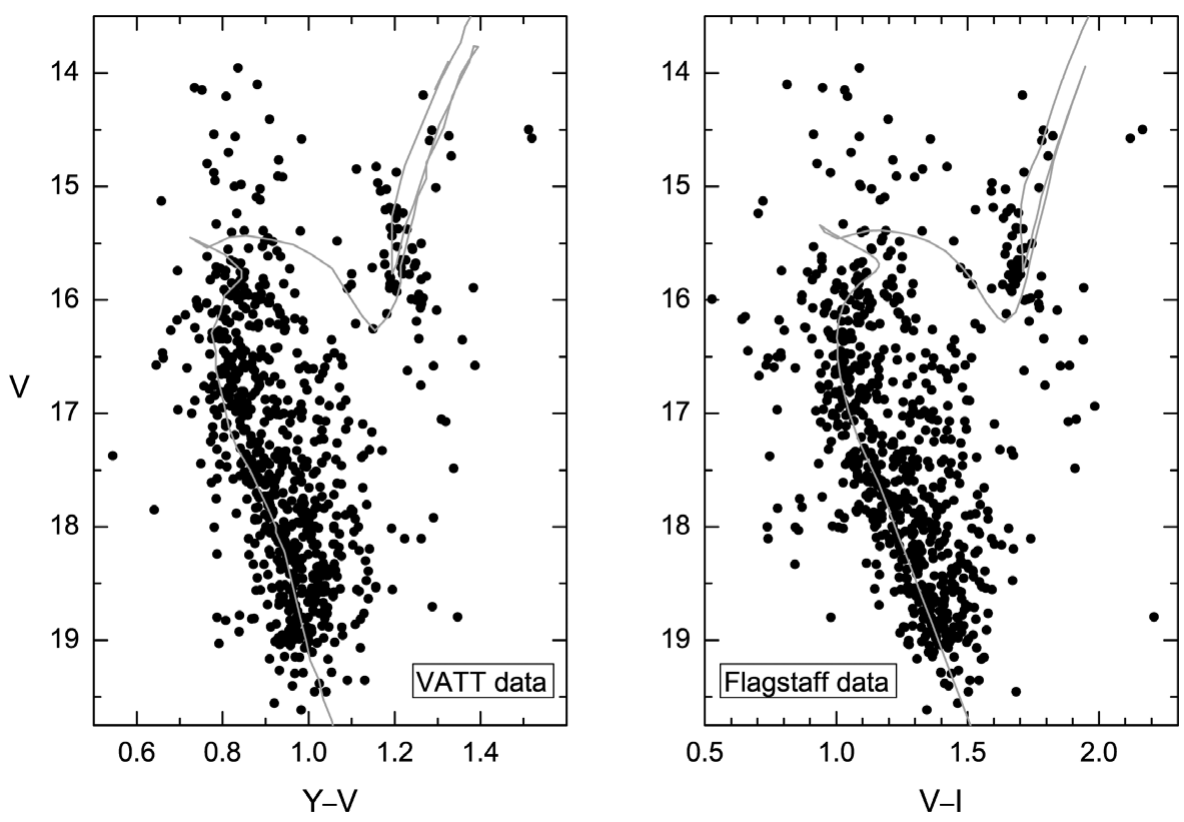

Figure 2. Uncleaned CMDs for stars common to the VATT and Flagstaff data sets. The Padova isochrones shown are for $Z=0.010, \log (t / \mathrm{yr})=9.0$, and $(m-M)_{V}=14.7 \mathrm{mag}$ (see Figure 3); taken for $V, Y-V$ from Bressan \& Tautvaišienè (1996) and for $V, V-I$ from Marigo et al. (2008).

were taken as final results. The methods applied give an accuracy for the spectral class on the order of \pm 1 decimal spectral subclass. The accuracy of $M_{V}$ depends on spectral type and varies from \pm 0.2 to $\pm 0.5 \mathrm{mag}$. To calculate $A_{V}$ from the derived color excesses $E(Y-V)$, a ratio of $R=4.16$ was used.

Metallicities were determined only for the red-giant stars, using the dereddened colors $P-X, X-Y$, and $P-Y$, calibrated in terms of $[\mathrm{Fe} / \mathrm{H}]$. Most main-sequence stars are of spectral types earlier than F0, i.e., they fall outside the limits of the Vilnius-system calibration in $[\mathrm{Fe} / \mathrm{H}]$.

The most probable photometric members of the cluster were selected by combining the following precepts:

(1) statistical removal of field stars using the $V_{0}, Q_{X Y Z}$ diagram;

(2) concentration of stars in the $A_{V}$ versus $(m-M)_{V}$ diagram;

(3) consistency of the classification results obtained from different methods.

Within $7^{\prime}$ from the cluster center, the number of statistically identified members down to $I=19.6 \mathrm{mag}$ amounts to $\sim 800$. However, combining all adopted criteria reduces the number of the most probable members to only 200. These stars were used to derive the main cluster parameters. By averaging the individual values we find

- the mean distance modulus $(m-M)_{0}=12.7 \pm 0.2 \mathrm{mag}$,

- the mean metallicity $[\mathrm{Fe} / \mathrm{H}]=0.3 \pm 0.1 \mathrm{dex}$ (only the red giants were used),

- the mean extinction $A_{V}=2.1 \mathrm{mag}$, with a clear indication of variable extinction ranging from 1.9 to $2.6 \mathrm{mag}$.

The CMDs of the probable members are shown in Figure 3, which give further evidence of the presence of nonuniform interstellar extinction in the direction of the cluster. Displayed in the $V_{0},(Y-V)_{0}$ diagram are the same stars as in the left panel, but corrected for individual reddening and extinction estimates. The scatter in the $V_{0},(Y-V)_{0}$ 

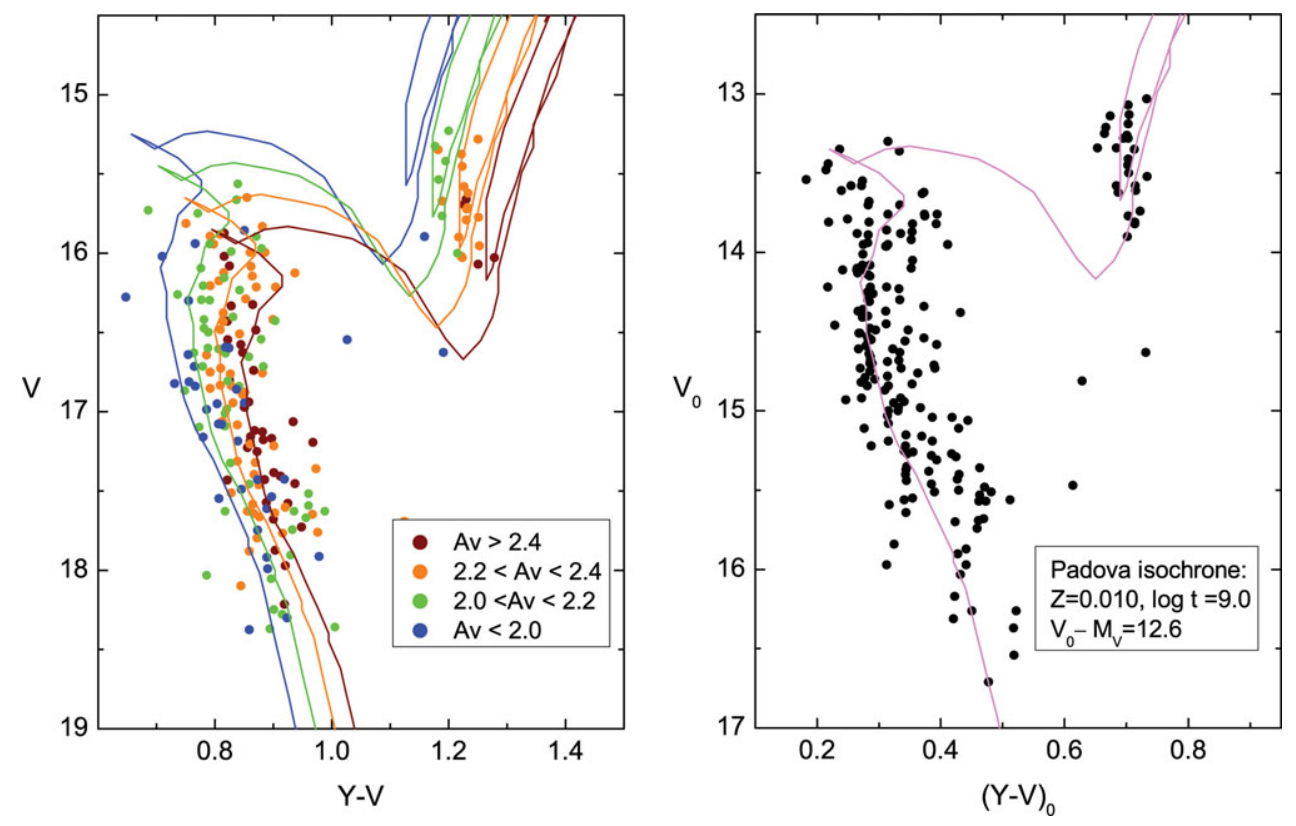

Figure 3. CMDs for probable photometric members, with the best-fitting isochrone shown: $Z=0.010, \log (t / \mathrm{yr})=9.0$, and $(m-M)_{0}=12.6 \mathrm{mag}$. (left) $V, Y-V$ diagram with the same isochrone adjusted for different values of $A_{V}$. (right) The same stars in the $V_{0},(Y-V)_{0}$ diagram.

diagram is, however, similar to that seen in the left panel, but the stars are more concentrated around the best-fitting isochrone. This $Z=0.010$ isochrone gives the following cluster parameters:

- a distance modulus $(m-M)_{0}=12.6 \mathrm{mag}$, or a distance of $3.3 \mathrm{kpc}$,

- an age of $1.0 \pm 0.15$ Gyr.

\section{Conclusion}

We conclude that IC 361 is a distant intermediate-age cluster located in, or just beyond, the Perseus spiral arm. Its mild metal deficiency is in agreement with the Galactic metallicity gradient. However, the presence of high and variable extinction, also confirmed by IRAS $100 \mu \mathrm{m}$ data, complicates a more detailed study of this beautiful open cluster.

\section{Acknowledgements}

JZ acknowledges support through the Lithuanian State Science and Studies Foundation grant S-17/2008.

\section{References}

Bressan, A. \& Tautvaišienè, G. 1996, BaltA, 5, 239

Laugalys, V., Kazlauskas, A., Boyle, R. P., Vrba, F. J., Philip, A. G. D., \& Straižys, V. 2004, Balt A, 13, 1

Marigo, P., Girardi, L., Bressan, A., Groenewegen, M. A. T., Silva, L., \& Granato, G. L. 2008, $A \& A, 482,883$

Piccirillo, J. \& Stein W. L. 1978, AJ, 83, 971 